\title{
28 Research Square \\ The Effect of the PARK16 rs11240572 Variant on Brain Structure in Parkinson's Disease
}

\section{Lu-yan Gu}

Zhejiang University

Shao-bing Dai

Zhejiang University

\section{Cheng Zhou}

Zhejiang University

\section{Ting Gao}

Zhejiang University

Jing-jing Wu

Zhejiang University

\section{Yi Fang}

Zhejiang University

Xiao-jun Guan

Zhejiang University School of Medicine Second Affiliated Hospital

\section{Tao Guo}

Zhejiang University

Ran Zheng

Zhejiang University

Chongyao Jin

Zhejiang University

\section{Xiao-jun Xu}

Zhejiang University School of Medicine Second Affiliated Hospital

\section{Zhe Song}

Zhejiang University School of Medicine Second Affiliated Hospital

Jun Tian

Zhejiang University School of Medicine Second Affiliated Hospital

\section{Xinzhen Yin}

Zhejiang University School of Medicine Second Affiliated Hospital

\section{Min-min Zhang}

Zhejiang University School of Medicine Second Affiliated Hospital

\section{Bao-rong Zhang}

Zhejiang University School of Medicine Second Affiliated Hospital 
Zhejiang University School of Medicine Second Affiliated Hospital

Jiali Pu ( $\sim$ jialipu@zju.edu.cn )

Zhejiang University School of Medicine Second Affiliated Hospital

\section{Research Article}

Keywords: Parkinson's disease, PARK16, rs11240572, structural MRI, voxel-based morphometry

Posted Date: June 24th, 2021

DOl: https://doi.org/10.21203/rs.3.rs-418680/v1

License: (c) (i) This work is licensed under a Creative Commons Attribution 4.0 International License. Read Full License

Version of Record: A version of this preprint was published at Brain Structure and Function on August 9th, 2021. See the published version at https://doi.org/10.1007/s00429-021-02359-9. 


\section{Abstract \\ Background}

Increasing evidence suggests that genetic factors play a key role in the development of Parkinson's disease (PD). The variant rs 11240572 in the PARK16 gene locus is strongly associated with PD. However, its effect on the pathogenesis of PD is yet to be clarified.

\section{Objective}

To explore the effect of the PARK16 rs11240572 variant on brain structure in PD patients.

\section{Methods}

A total of 51 PD patients were enrolled in the study and genotyped for the rs 11240572 variant. Clinical assessments and MRI scans were conducted across all participants. Voxel-based morphometry (VBM) was used to investigate gray matter volume (GMV) of the whole brain between these two groups. Correlation analysis was performed to identify the relationships between GMV and clinical features.

\section{Results}

There were 17 rs11240572-A variant carriers and 34 non-carriers, with no significant demographic differences between these two groups. Compared with non-carriers, rs11240572-A carriers showed increased GMV in left caudate nucleus and putamen, but decreased GMV in left superior temporal gyrus and supramarginal gyrus. In non-carriers, left basal ganglia GMV was positively correlated with UPDRS III $(r=0.365, p=0.040)$ and bradykinesia $(r=0.409, p=0.020)$, but negatively correlated with MMSE $(r=$ $-0.391, p=0.027)$. When reduced the sample size to the level of the carrier sample, we still found the same correlations in the non-carrier group. However, no correlation was found between the altered GMV and clinical features in the carriers.

\section{Conclusions}

Our study showed that the PARK16 rs11240572 variant affects the brain structure of patients with PD, especially in the basal ganglia and temporoparietal cortex. This indicated that this variant might play an important role in the pathogenesis of PD.

\section{Introduction}


Parkinson's disease (PD) is a common neurodegenerative disorder affecting about $1.7 \%$ of the population $\geq 65$ years old in China (Zhang et al.,2005). The main clinical manifestations of PD are motor symptoms, such as resting tremor, rigidity, bradykinesia and postural instability. In addition, non-motor symptoms such as paresthesia, autonomic dysfunction and cognitive impairment often occur in the early stages of the disease. Pathological changes in PD include the loss of dopaminergic neurons in the substantia nigra pars compacta and the aggregation of asynuclein in intracellular inclusion bodies (Homayoun, 2018). These neurons, projecting to the basal ganglia, are substantially damaged, causing a reduction in the dopamine levels of the striatum (Hu and Wang, 2016).

Although the pathogenesis of PD is still elusive, evidence suggests that genetic factors are closely related to PD susceptibility. Research has improved the understanding of PD genetics, especially as novel gene loci and disease-related SNPs in PD are progressively identified. Some mutational SNPs either protect against or aggravate the progression of the disease (Guo et al., 2015). The PARK16 locus, which is strongly associated with PD, was first identified by a genome-wide association study in a Japanese population (Satake et al., 2009). Subsequently, its role was confirmed in various regions, including Europe, China, Singapore, Chile and Malaysia, and also in the Jewish population (Simón-Sánchez et al., 2009; Tan et al., 2010; Liu et al., 2011; Mata et al., 2011; Ramirez et al., 2011; Gopalai et al., 2016). Within PARK16, rs11240572 is the variant most robustly associated with risk modulation of PD in a Chinese population (Tan et al., 2010). Subsequent studies demonstrated that rs11240572 were associated with PD risk reduction in Chinese population (Chang et al., 2013; Tan et al., 2010; Yan et al., 2011). However, the role of PARK16 in PD is still elusive. A recent longitudinal study demonstrated that rs11240572variant carriers showed greater motor function deterioration in PD compared with non-carriers (Deng et al., 2019), although its exact mechanism in PD remains unclear.

Neuroimaging is widely used in brain research as it is non-invasive. Voxel-based-morphometry (VBM) is a whole-brain, quantitative technique for characterizing global and regional cerebral volume, as well as differences of gray or white matter density in structural MRI (Good et al., 2001). Compared to traditional MRI measurements, which are based on regions of interest, VBM directly analyzes the original data without prior assumption, and quantitatively detects differences in brain tissue density. It is therefore not affected by subjective influences, such as the experience and diligence of the researcher. Its advantages are therefore that is automatic, comprehensive, objective and repeatable. Previous studies exploring the relationships between VBM changes and genetic factors in PD have yielded inconsistent conclusions (Brockmann et al., 2011; Vilas et al., 2016). Vilas et al. (2016) found either decreased basal ganglia gray matter volume or no volume difference in asymptomatic carriers with LRRK2 mutation compared with controls. However, Brockmann et al. (2011) found a volume decrease in symptomatic LRRK2 carriers (i.e. PD patients) - not in asymptomatic carriers. Additionally, previous studies have suggested that various SNPs, such as SNCA rs356219 (Burciu et al., 2018) and rs894278 (Zhang et al., 2019), are also involved in the modulation of brain functional activity. However, there are few studies exploring their direct effect on brain structure. 
In this study, we investigated the role of the PARK16 rs11240572 variant on the brain structure of PD patients in a Chinese cohort.

\section{Materials And Methods}

\section{Study participants}

A total of 51 PD patients were enrolled in this study, including 17 rs11240572-A variant carriers and 34 non-carriers. They were recruited at the Second Affiliated Hospital of Zhejiang University School of Medicine between April 2016 and July 2019. PD diagnosis was made by two experienced neurologists according to the Movement Disorder Society (MDS) Clinical Diagnostic Criteria for Parkinson's disease (Postuma et al., 2015). All participants underwent clinical evaluations and 3.0 Tesla MRI scans. The exclusion criteria were as follows: 1 ) a history of cerebrovascular injury, brain trauma and other neurodegenerative or psychiatric diseases; 2) contraindications for MRI such as metal implants, mental disorders, etc; and 3) refusal to sign informed consent. This study was approved by the ethics committee of the Second Affiliated Hospital of Zhejiang University School of Medicine and all participants gave informed consent prior to data collection and scaning.

\section{Clinical assessment}

A detailed personal and medical history was obtained from each participant, and neuropsychological examinations were conducted. PD severity and disease status were evaluated using the Unified Parkinson's Disease Rating Scale (UPDRS) and the Hoehn and Yahr ( $\mathrm{H}-\mathrm{Y})$ scale which obtained in the "drug-off " state (the patient has been off medication for at least 12 hours); and cognitive function was assessed using a Mini-Mental State Examination (MMSE). The scores for tremor, rigidity, bradykinesia, and posture/gait were calculated by adding the scores from items $20+21$, item 22, items $23+24+25+$ $26+31$, and items $27+28+29+30$ of UPDRS III, respectively.

\section{Genotyping}

Genomic DNA of all participants was extracted from peripheral blood samples using standard procedures and assessed with a Qubit 3.0 Fluorometer (Life Invitrogen). PCR and direct sequencing were used to genotype the PARK16 rs11240572 variant. PD patients were classified as carriers if they were found to have the PARK16 rs11240572 A allele, including AC and AA, or non-carriers if they had the PARK16 rs11240572 homozygous C allele.

\section{MRI acquisition}

All subjects were scanned using a 3.0 Tesla MRI scanner (GE Discovery 750) at the Department of Radiology, Second Affiliated Hospital, School of Medicine, Zhejiang University. The 3D T1 weighted images were acquired using a Fast Spoiled Gradient Recalled sequence: echo time $=3.036 \mathrm{~ms}$; repetition time $=7.336 \mathrm{~ms}$; inversion time $=450 \mathrm{~ms}$; flip angle $=11$ degrees; field of view $=260 \times 260 \mathrm{~mm}^{2} ;$ matrix $=$ 
$256 \times 256$; in-plain resolusion $=1 \times 1$; slice thickness $=1.2 \mathrm{~mm}$; 196 sagittal slices. The precise voxel size $=1 \times 1 \times 1.2 \mathrm{~mm}$, and the voxel were almost isotropic.

\section{Voxel-based morphometry}

MR images of the brain were preprocessed using the Computational Anatomy Toolbox (CAT12: http://dbm.neuro.uni-jena.de/cat12/) implemented within Statistical Parametric Mapping 12 software (SPM12, http://www.fil.ion.ucl.ac.uk/spm). Data were preprocessed with CAT12 (http://www.neuro.unijena.de/cat12/CAT12-Manual.pdf) as follows:1) the original T1 images were normalized to a template space and segmented into gray matter (GM), white matter (WM) and cerebrospinal fluid (CSF); 2) the quality of the image was manually checked to maintain sample homogeneity. The unsmoothed segmentations were used to provide more anatomical detail; 3 ) total intracranial volume (TIV) was estimated to correct individual brain size during statistical analysis; 4) all segmented GM images were smoothed with a Gaussian kernel of $6 \mathrm{~mm}$ full-width-half-maximum (FWHM).

\section{Statistical analysis}

Demographic and clinical data were analyzed using SPSS version 23.0 (IBM, Chicago, IL, USA). Statistical charts were produced by GraphPad Prism 7.0a (GraphPad Inc., San Diego, CA, USA). The results were expressed as mean \pm standard deviation (SD) for continuous variables and as frequencies for the categorical variables. The Kolmogorov-Smirnov test was used to identify the normality of variable distribution. Independent t-test was used to compare normally distributed continuous variables, and the Mann-Whitney $U$ test was used to compare continuous variables that were not normally distributed. The chi-squared test was used for the comparison of categorical variables. Spearman's rank correlation analysis was performed to evaluate the correlations between basal ganglia GMV, temporoparietal cortex GMV and UPDRS III, tremor, rigidity, bradykinesia, posture/gait, MMSE scores, respectively. Age and sex were entered as covariates in the analysis due to their potential influence. A p value $<0.05$ was considered significant.

Image analysis was performed in the Data Processing \& Analysis for Brain Imaging toolbox (Yan et al., 2016). Two-sample t-test was conducted to compare the GMV between the two groups of participants. In order to balance the type I and type II errors in the multivoxel comparison(Lieberman and Cunningham 2009), a conservative threshold with Gaussian Random Field correction of voxel $p=0.001$ and cluster $p=$ 0.05 , and a preliminary threshold with Gaussian Random Field correction of voxel $p=0.005$ and cluster $p$ $=0.05$ were employed. Age, sex, and TIV were incorporated as covariates.

\section{Results}

\section{Demographic and clinical features}

The demographic and clinical features are summarized in Table 1. There were no significant differences between carriers and non-carriers in clinical characteristics including age, gender, years of education, 
disease duration, scores of UPDRS, UPDRS I, UPDRS II, UPDRS III or UPDRS IV, tremor, rigidity, bradykinesia, posture/gait, $\mathrm{H}-\mathrm{Y}$ scale stage or MMSE.

\section{Group differences in VBM}

After taking sex, age and TIV as covariates, carriers showed increased GMV recognized in the left basal ganglia (mainly in caudate nucleus and putamen); while decreased GMV was detected in the left temporoparietal cortex (mainly in superior temporal gyrus and supramarginal gyrus) compared with noncarriers(threshold GRF of voxel $P=0.005$, cluster $P=0.05$, the corrected cluster size was greater than 861 voxels). The results of the two-sample T-test are shown in Figure 1 and Table 2. However, these GMV differences did not survive after applying a stricter statistical threshold of voxel $\mathrm{P}=0.001$, cluster $\mathrm{P}=0.05$

\section{Correlation analysis}

Correlation analyses are summarized in Figure 2. We found that the GMV of the left basal ganglia was positively correlated with UPDRS III $(r=0.365, p=0.040)$ and bradykinesia $(r=0.409, p=0.020)$, but negatively correlated with MMSE scores $(r=-0.391, p=0.027)$ in non-carriers after taking age and gender into account. After matching the sample size to the level of the carrier sample, we still found the same correlations in the non-carrier group (UPDRS III, $r=0.594, p=0.020$; bradykinesia, $r=0.606, p=0.017$; MMSE, $r=-0.516, p=0.049$; see Figure 3). No correlation was found between the altered GMV and clinical features in carriers.

\section{Discussion}

We believe that this is the first study to investigate the effect of the PARK16 rs11240572 variant on the GMV of the whole brain in PD patients by VBM analysis. Our results showed that patients with the variant had a different brain structure to a control group in a Chinese PD cohort. The differences of brain structure were located at basal ganglia and temporoparietal cortex, hinting that it might play a key role in the pathogenesis of PD.

PARK16 is located on chromosome 1q32, and seven of its SNPs (rs16856139, rs823128, rs823122, rs947211, rs823156, rs708730 and rs11240572) were found to be risk factors for PD in a Japanese cohort (Satake et al., 2009). However, subsequent studies demonstrated that some SNPs, including rs11240572, were in fact associated with PD risk reduction in a Chinese population (Chang et al., 2013; Tan et al., 2010; Yan et al., 2011). The SNP rs11240572 is located in the peptidase M20-domaincontaining protein 1 (PM20D1) gene, which is associated with metal ion binding and peptidase activity (Kimura et al., 2006). Brain iron deposition is closely related to PD, as the accumulation of iron in the substantia nigra has been recognized as a major characteristic of PD (Guan et al., 2017; Sanchez-Mut et al., 2018). However, whether rs 11240572 also affects iron homeostasis in the brain need to be further elucidated. 
Our study found that PARK16 rs11240572-A variant carriers had increased GMV of basal ganglia, along with better motor and cognitive scores. These results suggest that the rs11240572-A variant may slow down the progression of $\mathrm{PD}$, consistent with previous reports suggesting that it has a protective function (Chang et al., 2013; Tan et al., 2010; Yan et al., 2011). The pathogenesis of PD involves the basal ganglia, including the caudate nucleus and putamen, resulting in impaired movement control (Fang et al. 2020) as well as poor cognition such as impaired learning, planning, memory, and emotion (Obeso et al., 2008; Gao et al., 2017). Previous studies have found that PD patients have a variable GMV in the putamen, caudate nucleus, cerebellum and cerebral cortex. Brockmann et al. (2011) reported a decreased basal ganglia GMV, especially in the putamen of LRRK2 mutation patients. Similarly, Reetz et al. (2009) showed that subjects with a parkin gene mutation had decreased basal ganglia GMV. In contrast, two studies (Burciu et al., 2018; Vilas et al., 2016) found no change in the brain structure of patients with either a LRRK2 mutation or a SNCA variant. We use a preliminary threshold with GRF correction of voxel $p=0.005$ and cluster $p=0.05$. Previous study has reported positive results of VBM using volumetric threshold of $p<$ 0.005 and cluster level of $p<0.05$. (Engelhardt, Boulat et al. 2020). No findings survived with a threshold of voxel $p<0.001$ and cluster $p<0.05$.

In the non-carrier group, we also found left basal ganglia GMV was positively correlated with UPDRS III and bradykinesia, but negatively correlated with MMSE using correlation analysis. Several studies has found similar results and explained these phenomena as plasticity compensation of neurons (Binkofski et al. 2007, Brockmann et al. 2011).Changes in GM signal extracted from MRI images can reflect various processes, such as changes in the number of synapses, the number of glial cells, the number of neurons, dendritic structure, vasculature, blood volume and circulation, and myelination. (Spruston et al.,2008, Hoekzema et al., 2017). In the structure of neurons, dendrites are key sites for synaptic integration and neuronal connectivity in the brain. Kassem et al.(2013) demonstrated that grey matter loss determined by MRI is primarily due to loss of dendrites and their synapses in stressed mice. Therefore, we hypothesized that the increase in GMV in the basal ganglia, indicating the upregulation of functional dendrites and synapses, may be a compensatory alteration secondary to the dopamine depletion in this region in a special disease stage, and probably in the following years the volume would be decreased with the exhaustion of the ability to maintain the brain function. Of course, there are many studies demonsrtated that the smaller the gray matter volume is, the greater the damage is, and the worse the disease is. However, no correlation was found between the altered GMV and clinical features in rs11240572-A carriers. Inconsistencies in results may be due to differences in sample size, research group, and disease stage. In order to figure out the effect of group size on our correlation result, we reduced the sample size to the level of the carrier sample and still found the same correlations in the non-carrier group, which strengthen the speculation about a meaningful group difference between correlation findings of both groups.

Previous studies suggested that PD patients with mild cognitive impairment might suffer from atrophy of the temporal and parietal areas (Pereira et al., 2014; Zhang et al., 2018). In contrast, Binudo et al. (2013) demonstrated that mild cognitive impairment in PD was associated with significant regional thickening in the left temporal-occipital lobe and the thickening has been explained by compensatory neuroplasticity. 
The association between temporal GMV and cognition impairment in PD is therefore still controversial. Our study showed decreased GMV of both the left superior temporal gyrus and supramarginal gyrus was negatively associated with cognitive function in rs11240572-A non-carriers, which might indicate the compensatory neuroplasticity.

In addition, instead of bilaterally symmetrical GMV alterations, our study showed differences in the left basal ganglia and left temporoparietal cortex between the two groups of patients. Recently, Garrido et al. (2020) reported the left hemispheric predominance of nigrostriatal deficit in right-handed PD. Thus, it might be account of the fact that most of our subjects were right-handed or the limited sample size. Further studies will be needed to address the issues.

There were several limitations in the present study. First, because of the difficulty in acquiring genetic and imaging data from the same participants, the sample size recruited in the present study is relatively small. We subdivided the patients according to their genetic profiles, which may lead to the different group sizes. Due to the limited sample size, the results should be interpreted cautiously and larger studies are needed to validate and further our findings. Secondly, participants were only estimated by VBM, which can lack accuracy. Therefore a variety of neuroimaging approaches, such as functional MRI and quantitative susceptibility mapping, should be incorporated into future work to elucidate the genetic effect in PD. In spite of these limitations, our study provides novel clues for future efforts to explore the possible pathological mechanism underlying PARK16-related PD.

\section{Conclusion}

In conclusion, the present study showed that PARK16 rs11240572 variant carriers have altered GMV in the left basal ganglia and left temporoparietal cortex, indicating that this SNP might play an important role in the pathogenesis of PD. Further research on the function and mechanism of the PARK16 locus is required.

\section{Declarations}

Financial Disclosure/Conflict of Interest: The authors declare no conflicts of interest.

Funding: This work was supported by the National Natural Science Foundation of China's Major Regional International Cooperation Project [No.81520108010], the 13th Five-year Plan for National Key Research and Development Program of China [No.2016YFC1306600], the National Natural Science Foundation of China [No.81771216 and No.81971577], the Key Research and Development Program of Zhejiang Province [No.2020C03020], the Natural Science Foundation of Zhejiang Provincial (No.LY18H090003) and the Project funded by China Postdoctoral Science Foundation [No.2019M662082].

\section{References}


1. Binkofski, F.,Reetz, K.,Gaser, C.,Hilker, R.,Hagenah, J.,Hedrich, K.,(2007). Morphometric fingerprint of asymptomatic Parkin and PINK1 mutation carriers in the basal ganglia.

Neurology. 2007 Aug 28;69(9):842-50.https://doi: 10.1212/01.wnl.0000267844.72421.6c.

2. Biundo, R., Calabrese, M., Weis, L., Facchini, S., Ricchieri, G., Gallo, P., et al. (2013). Anatomical correlates of cognitive functions in early Parkinson's disease patients. PloS one, 8: e64222. https://doi.org/10.1371/journal.pone.0064222

3. Brockmann, K., Groger, A., Di Santo, A., Liepelt, I., Schulte, C., Klose, U., et al. (2011). Clinical and brain imaging characteristics in leucine-rich repeat kinase 2-associated PD and asymptomatic mutation carriers. Mov Disord, 26: 2335-42. https://doi.org/10.1002/mds.23991

4. Burciu, R. G., Seidler, R. D., Shukla, P., Nalls, M. A., Singleton, A. B., Okun, M. S., et al. (2018). Multimodal neuroimaging and behavioral assessment of alpha-synuclein polymorphism rs356219 in older adults. Neurobiol Aging, 66: 32-39. https://doi.org/10.1016/j.neurobiolaging.2018.02.001

5. Chang, K. H., Chen, C. M., Chen, Y. C., Lyu, R. K., Chang, H. S., Ro, L. S., et al. (2013). Association between PARK16 and Parkinson's disease in the Han Chinese population: a meta-analysis. Neurobiol Aging, 34: 2442.e5-9. https://doi.org/10.1016/j.neurobiolaging.2013.04.028

6. Deng, X., Xiao, B., Allen, J. C., Ng, E., Foo, J. N., Lo, Y. L., et al. (2019). Parkinson's disease GWASlinked Park16 carriers show greater motor progression. J Med Genet, 56: 765-68. https://doi.org/10.1136/jmedgenet-2018-105661

7. Engelhardt, C., B. Boulat, M. Czisch and M. V. Schmidt (2020). "Lack of FKBP51 Shapes Brain Structure and Connectivity in Male Mice." J Magn Reson Imaging.

8. Fang, E., Ann, C.N., Maréchal, B., Lim, J.X., Tan, S.Y.Z., Li, H., et al. (2020). Differentiating Parkinson's disease motor subtypes using automated volume-based morphometry incorporating white matter and deep gray nuclear lesion load. J Magn Reson Imaging, 51: 748-56. https://doi.org/10.1002/jmri.26887

9. Gao, Y., Nie, K., Huang, B., Mei, M., Guo, M., Xie, S., et al. (2017). Changes of brain structure in Parkinson's disease patients with mild cognitive impairment analyzed via VBM technology, Neurosci Lett, 658: 121-32. https://doi.org/10.1016/j.neulet.2017.08.028

10. Garrido, A., Iranzo, A., Stefani, A., Serradell, M., Muñoz-Lopetegi, A., Marrero, P., et al. (2020). Lack of Asymmetry of Nigrostriatal Dopaminergic Function in Healthy Subjects, Mov Disord, https://doi.org/10.1002/mds.28019

11. Good, C. D., Johnsrude, I. S., Ashburner, J., Henson, R. N., Friston, K. J., Frackowiak, R. S. (2001). A voxel-based morphometric study of ageing in 465 normal adult human brains. Neuroimage, 14: 21 36. https://doi.org/10.1006/nimg.2001.0786

12. Gopalai, A. A., Ahmad-Annuar, A., Li, H.H., Zhao, Y., Lim, S.Y., Tan ,A. H., et al. (2016). PARK16 is associated with PD in the Malaysian population, Am J Med Genet B Neuropsychiatric Genet, 171: 839-47. https://doi.org/10.1002/ajmg.b.32454

13. Guan, X., Xu, X., Zhang, M.M. (2017). Region-Specific Iron Measured by MRI as a Biomarker for Parkinson's Disease, Neurosci Bull, 33: 561-67. https://doi.org/10.1007/s12264-017-0138-X 
14. Guo, J. F., Li, K., Yu, R. L., Sun, Q. Y., Wang, L., Yao, L. Y., et al. (2015). Polygenic determinants of Parkinson's disease in a Chinese population, Neurobiol Aging, 36: 1765.e1-65.e6. https://doi.org/10.1016/j.neurobiolaging.2014.12.030

15. Hoekzema, E.,Barba-Müller, E.,Pozzobon, C.,Picado, M.,Lucco, F.,García-García, D.,et al. (2017). Pregnancy leads to long-lasting changes in human brain structure,Nat Neurosci. 2017 Feb;20(2):287296. doi: https://10.1038/nn.4458. Epub 2016 Dec 19.

16. Homayoun, H. (2018). Parkinson Disease, Ann Intern Med, 169: ITC33-ITC48. https://doi.org/10.7326/AITC201809040

17. Hu, Q., Wang, G. (2016). Mitochondrial dysfunction in Parkinson's disease, Transl Neurodegener, 5: 14. https://doi.org/10.1186/s40035-016-0060-6

18. Kassem, M. S., Lagopoulos, J.,Stait-Gardner, T.,Price, W. S.,Chohan, T. W.,Arnold, J. C., et al. (2013). Stress-induced grey matter loss determined by MRI is primarily due to loss of dendrites and their synapses.Mol Neurobiol, 47(2):645-61. https://doi.org/10.1007/s12035-012-8365-7

19. Kimura, K., Wakamatsu, A., Suzuki, Y., Ota, T., Nishikawa, T., Yamashita, R., et al. (2006). Diversification of transcriptional modulation: large-scale identification and characterization of putative alternative promoters of human genes. Genome Res, 16: 55-65. https://doi.org/10.1101/gr.4039406

20. Lieberman, M. D.,Cunningham, W. A. (2009). Type I and Type II error concerns in fMRI research: rebalancing the scale. Soc Cogn Affect Neurosci, 4(4):423-8. doi: 10.1093/scan/nsp052.

21. Liu, X.M., Cheng R., Verbitsky, M., Kisselev, S., Browne, A., Mejia-Sanatana, H., et al. (2011). Genomewide association study identifies candidate genes for Parkinson's disease in an Ashkenazi Jewish population. BMC Med Genet, 12: 104. https://doi.org/10.1186/1471-2350-12-104

22. Mata, I. F., Yearout, D., Alvarez, V., Coto, E., de Mena, L., Ribacoba, R., et al. (2011). Replication of MAPT and SNCA, but not PARK16-18, as susceptibility genes for Parkinson's disease. Mov Disord, 26: 819-23. https://doi.org/10.1002/mds.23642

23. Obeso, J.A., Rodríguez-Oroz, M.C., Benitez-Temino, B., Blesa, F.J., Guridi, J., Marin, C., et al. (2008). Functional organization of the basal ganglia: therapeutic implications for Parkinson's disease. Mov Disord, 23 Suppl: S548-59. https://doi.org/10.1002/mds.22062

24. Pereira, J.B., Svenningsson, P., Weintraub, D., Brønnick, K., Lebedev, A., Westman, E., et al. (2014). Initial cognitive decline is associated with cortical thinning in early Parkinson disease. Neurology, 82: 2017-25. https://doi.org/10.1212/wnl.0000000000000483

25. Postuma, R. B., Berg, D., Stern, M., Poewe, W., Olanow, C. W., Oertel, W., et al. (2015). MDS clinical diagnostic criteria for Parkinson's disease. Mov Disord, 30: 1591-601. https://doi.org/10.1002/mds.26424

26. Ramirez, A., Ziegler, A., Winkler, S., Kottwitz, J., Giesen, R., Diaz-Grez, F., et al. (2011). Association of Parkinson disease to PARK16 in a Chilean sample. Parkinsonism Relat Disord, 17: 70-1. https://doi.org/10.1016/j.parkreldis.2010.09.002 
27. Reetz, K., Gaser, C., Klein, C., Hagenah, J., Büchel, C., Gottschalk, S., et al. (2009). Structural findings in the basal ganglia in genetically determined and idiopathic Parkinson's disease. Mov Disord, 24: 99103. https://doi.org/10.1002/mds.22333

28. Sanchez-Mut, J V., Heyn, H., Silva, B.A., Dixsaut, L., Garcia-Esparcia, P., Vidal, E., et al. (2018). PM20D1 is a quantitative trait locus associated with Alzheimer's disease. Nat Med, 24: 598-603. https://doi.org/10.1038/s41591-018-0013-y

29. Satake, W., Nakabayashi, Y., Mizuta, I., Hirota, Y., Ito, C., Kubo, M., et al. (2009). Genome-wide association study identifies common variants at four loci as genetic risk factors for Parkinson's disease. Nat Genet, 41: 1303-07. https://doi.org/10.1038/ng.485

30. Simón-Sánchez, J., Schulte, C., Bras, J. M., Sharma, M., Gibbs, J. R., Berg, D., et al. (2009). Genomewide association study reveals genetic risk underlying Parkinson's disease. Nat Genet, 41: 1308-12. https://doi.org/10.1038/ng.487

31. Spruston, N. (2018). Pyramidal neurons: dendritic structure and synaptic integration. Nat Rev Neurosci. 2008 Mar;9(3):206-21. https://doi: 10.1038/nrn2286.

32. Tan, E. K., Kwok, H. H., Kwok, H. K., Tan, L. C., Zhao, W. T., Prakash, K. M., et al. (2010). Analysis of GWAS-linked loci in Parkinson disease reaffirms PARK16 as a susceptibility locus. Neurology, 75 : 508-12. https://doi.org/10.1212/WNL.0b013e3181 eccfcd

33. Vilas, D., Segura, B., Baggio, H. C., Pont-Sunyer, C., Y. Compta, Valldeoriola, F., Marti, M. J., et al. (2016). Nigral and striatal connectivity alterations in asymptomatic LRRK2 mutation carriers: A magnetic resonance imaging study. Mov Disord, 31: 1820-28. https://doi.org/10.1002/mds.26799

34. Yan, C. G., Wang, X. D., Zuo, X. N., Zang, Y. F. (2016). DPABI: Data Processing \& Analysis for (RestingState) Brain Imaging, Neuro in formatics, 14: 339-51. https://doi.org/10.1007/s12021-016-9299-4

35. Yan, Y.P., Mo, X.Y., Tian, J., Zhao, G.H., Yin, X.Z., Jin, F.Y., et al. (2011). An association between the PARK16 locus and Parkinson's disease in a cohort from eastern China. Parkinsonism Relat Disord, 17: 737-39. https://doi.org/10.1016/j.parkreldis.2011.07.012

36. Zhang, K., Tang, Y., Meng, L., Zhu, L., Zhou, X., Zhao, Y., et al. (2019). The Effects of SNCA rs894278 on Resting-State Brain Activity in Parkinson's Disease. Front Neurosci, 13: 47. https://doi.org/10.3389/fnins.2019.00047

37. Zhang, L, Wang, M., Sterling, N.W., Lee, E.Y., Eslinger, P.J., Wagner, D., et al. (2018). Cortical Thinning and Cognitive Impairment in Parkinson's Disease without Dementia. IEEE/ACM Trans Comput Biol Bioinform, 15: 570-80. https://doi.org/10.1109/tcbb.2015.2465951

38. Zhang, Z.X., Roman, G.C., Hong, Z., Wu, C.B., Qu, Q.M., Huang, J.B., et al. (2005). Parkinson's disease in China: prevalence in Beijing, Xian, and Shanghai. Lancet, 365: 595-7. https://doi.org/10.1016/s0140-6736(05)17909-4

\section{Tables}

Table 1: Clinical data between rs11240572-A carriers and non-carriers among PD patients 


\begin{tabular}{|llcl|}
\hline Characteristics & A carriers(n=17) & A non-carriers(n=34) & $P$ \\
\hline Gender (male/female) & $9 / 8$ & $14 / 20$ & 0.426 \\
\hline Age & $60.39 \pm 12.58$ & $61.74 \pm 8.73$ & 0.655 \\
\hline Education & $8.88 \pm 5.23$ & $7.53 \pm 5.44$ & 0.401 \\
\hline Disease duration & $5.87 \pm 4.55$ & $5.37 \pm 5.32$ & 0.484 \\
\hline UPDRS score & $30.53 \pm 15.90$ & $35.15 \pm 16.29$ & 0.332 \\
\hline UPDRS I score & $1.41 \pm 1.58$ & $1.82 \pm 1.90$ & 0.517 \\
\hline UPDRS II score & $8.06 \pm 5.07$ & $9.09 \pm 6.00$ & 0.547 \\
\hline UPDRS III score & $19.76 \pm 10.63$ & $22.94 \pm 11.40$ & 0.368 \\
\hline UPDRS VI score & $1.29 \pm 1.40$ & $1.29 \pm 1.53$ & 0.900 \\
\hline Tremor score & $3.59 \pm 2.98$ & $3.29 \pm 2.69$ & 0.800 \\
\hline Rigidity score & $4.71 \pm 3.20$ & $5.65 \pm 3.98$ & 0.493 \\
\hline Bradykinesia score & $7.41 \pm 4.98$ & $9.50 \pm 5.63$ & 0.262 \\
\hline Posture/gait score & $2.88 \pm 1.22$ & $3.12 \pm 2.41$ & 0.847 \\
\hline HY scale & $2.27 \pm 0.47$ & $2.24 \pm 0.53$ & 0.720 \\
\hline MMSE & $27.65 \pm 2.60$ & $25.88 \pm 4.80$ & 0.220 \\
\hline
\end{tabular}

Data are shown as the mean \pm standard deviation.

"A carriers" represents the participants with at least one rs11240572 A allele;

"A non-carriers" represents the participants without rs11240572 A alleles.

PD, Parkinson's disease; UPDRS, Unified Parkinson's disease rating scale; HY, Hoehn and Yahr, MMSE, Mini-Mental State Examination;

Table 2: Anatomical regions of significant GMV alterations between rs11240572-A carriers and noncarriers of PD patients 


\begin{tabular}{|c|c|c|c|c|c|c|}
\hline \multirow[t]{2}{*}{ Regions (AAL) } & \multirow[t]{2}{*}{ Side } & \multicolumn{3}{|c|}{ Peak MNI coordinate } & \multirow[t]{2}{*}{ t-statistics } & \multirow[t]{2}{*}{ Cluster size } \\
\hline & & $X$ & $\mathbf{Y}$ & $\mathbf{Z}$ & & \\
\hline Cluster1 & & -4.5 & 18 & 10.5 & 4.26 & 1009 \\
\hline Caudate nucleus & $\mathrm{L}$ & & & & & 442 \\
\hline Putamen & $\mathrm{L}$ & & & & & 208 \\
\hline Cluster2 & & -45 & -36 & 7.5 & -4.71 & 1775 \\
\hline Superior temporal gyrus & $\mathbf{L}$ & & & & & 794 \\
\hline Supramarginal gyrus & $\mathrm{L}$ & & & & & 593 \\
\hline
\end{tabular}

Cluste1: Left basal ganglia; Cluster2: Left temporoparietal cortex

GMV, gray matter volume; PD, Parkinson's disease; L, Left;MNI, Montreal Neurological Institute; AAL, Automated Anatomic Labeling

Figures 


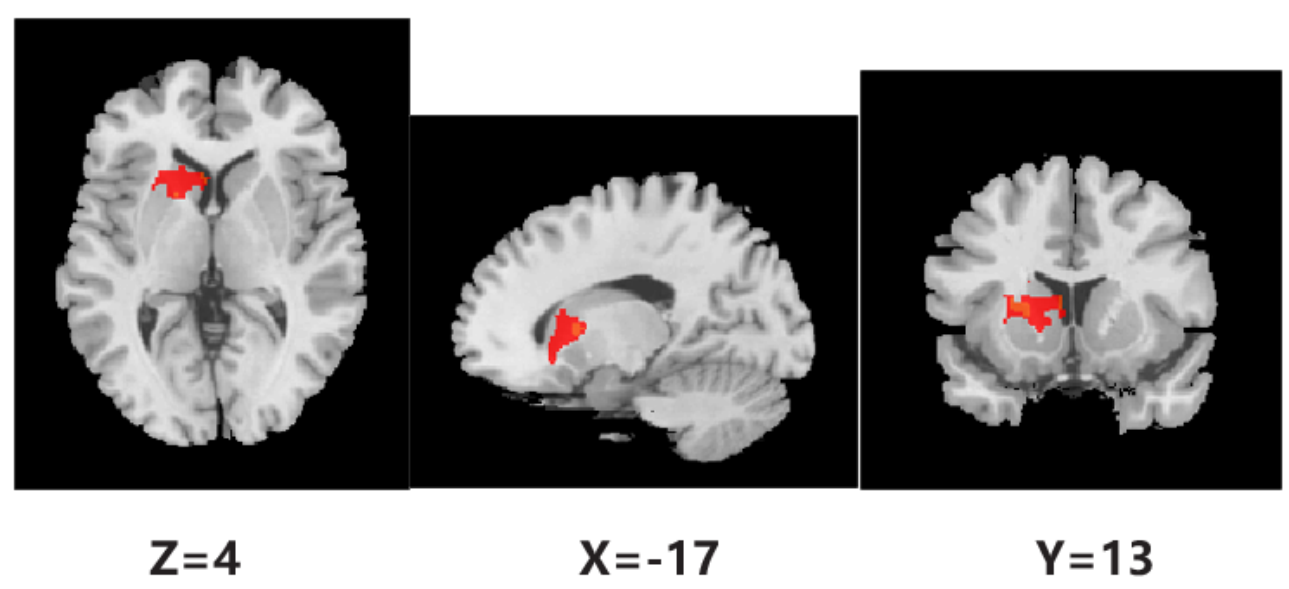

\section{Cluster1 (left caudate nucleus and putamen)}

rs11240572_A carriers - non-carriers

$\mathbf{L}$

$\mathbf{R}$

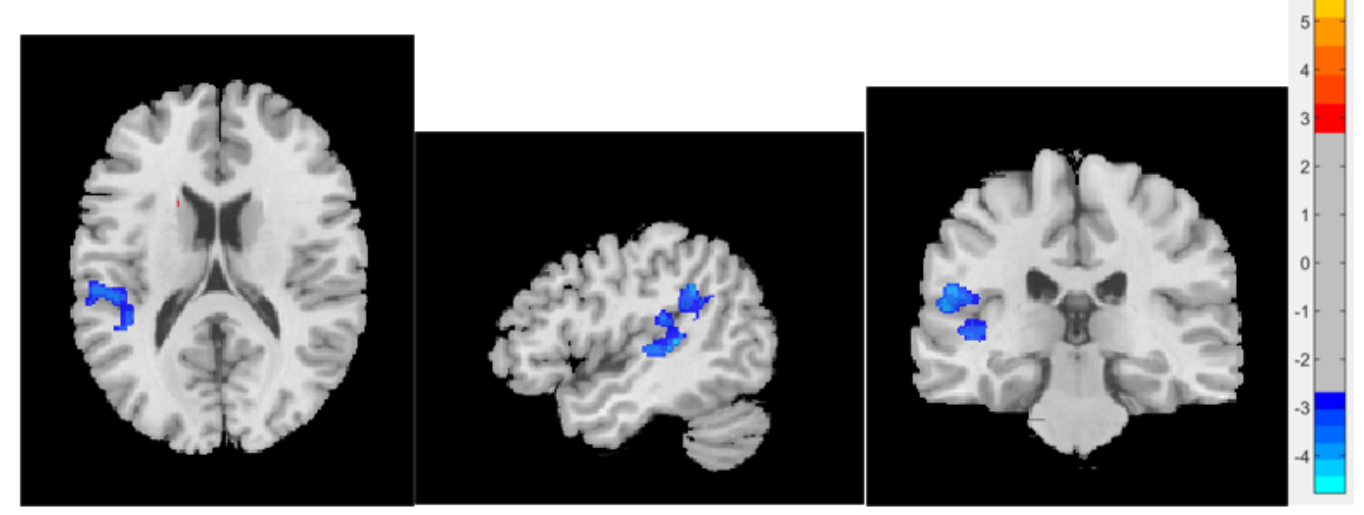

$$
\mathrm{Z}=7 \quad \mathrm{X}=-45 \quad \mathrm{Y}=-36
$$

Cluster2 (left superior temporal gyrus and supramarginal gyrus) rs11240572_A carriers - non-carriers

\section{Figure 1}

Anatomical regions of significant GMV alterations between rs11240572-A carriers and non-carriers. PARK16 rs11240572-A carriers have increased GMV in the left caudate nucleus and putamen, and decreased GMV in the left superior temporal gyrus and supramarginal gyrus compared with non- carriers. 

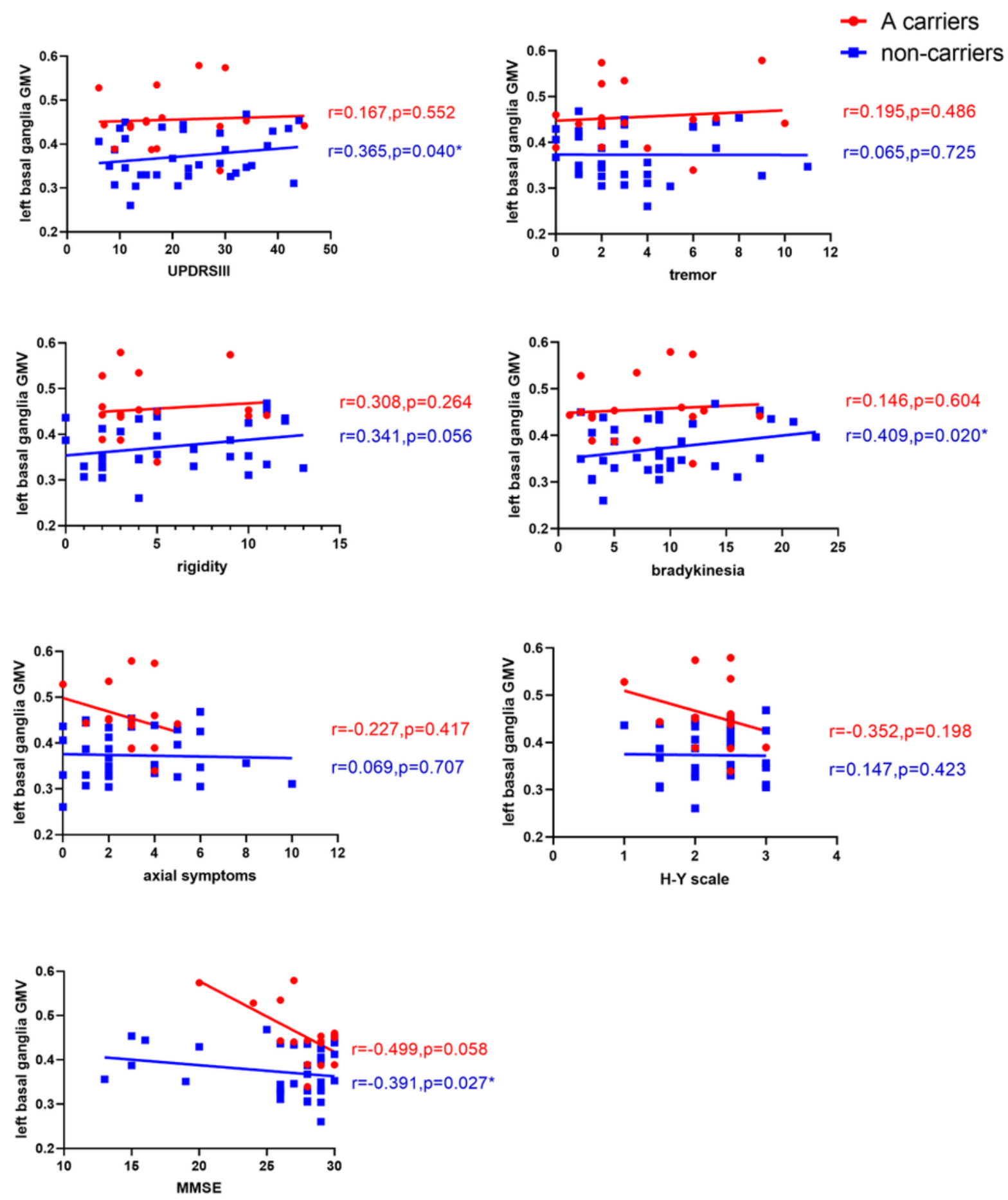

\section{Figure 2}

Correlation analysis. In rs 11240572 variant non-carriers, left basal ganglia GMV is positively correlated with UPDRS III $(r=0.365, p=0.040)$ and bradykinesia $(r=0.409, p=0.020)$, while negative correlation with MMSE scores is observed $(r=-0.391, p=0.027)$ UPDRS, Unified Parkinson's disease rating scale III; MMSE, Mini-Mental State Examination. 

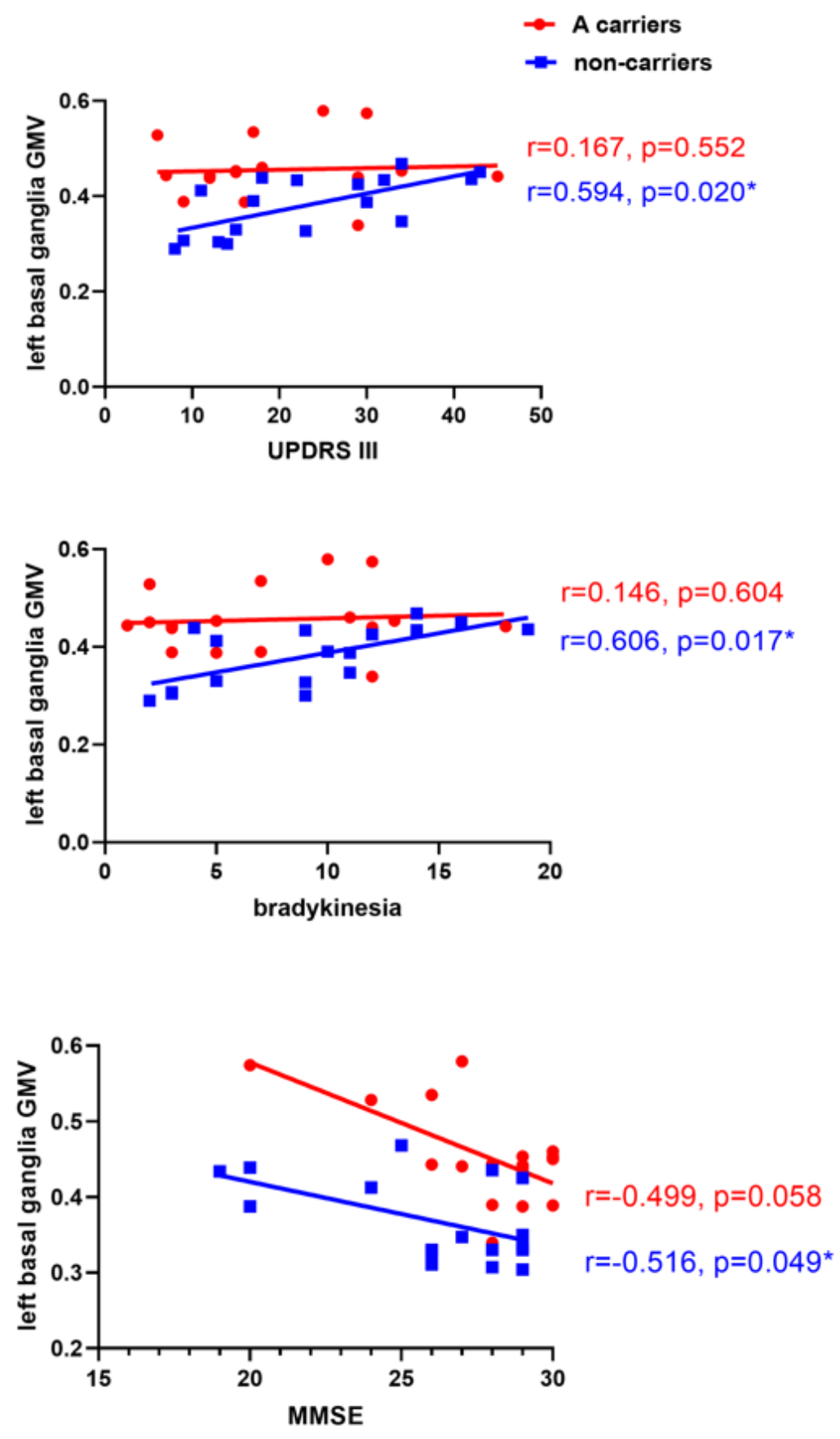

\section{Figure 3}

Correlation analysis. After matching the sample size of the non-carrier to the level of the carrier sample. In rs11240572 variant non-carriers, left basal ganglia GMV is still positively correlated with UPDRS III $(r=0.594, p=0.020)$ and bradykinesia $(r=0.606, p=0.017)$, while negative correlation with MMSE scores is observed $(r=-0.516, p=0.049)$ UPDRS, Unified Parkinson's disease rating scale III; MMSE, Mini-Mental State Examination. 